out well because they made predictions that survived testing. Yet numerous historical examples point to how, in the absence of adequate data, elegant and compelling ideas led researchers in the wrong direction, from Ptolemy's geocentric theories of the cosmos to Lord Kelvin's 'vortex theory' of the atom and Fred Hoyle's perpetual steady-state Universe.

The consequences of overclaiming the significance of certain theories are profound - the scientific method is at stake (see go.nature.com $/ \mathrm{hh} 7 \mathrm{~mm} 6$ ). To state that a theory is so good that its existence supplants the need for data and testing in our opinion risks misleading students and the public as to how science should be done and could open the door for pseudoscientists to claim that their ideas meet similar requirements.

What to do about it? Physicists, philosophers and other scientists should hammer out a new narrative for the scientific method that can deal with the scope of modern physics. In our view, the issue boils down to clarifying one question: what potential observational or experimental evidence is there that would persuade you that the theory is wrong and lead you to abandoning it? If there is none, it is not a scientific theory.

Such a case must be made in formal philosophical terms. A conference should be convened next year to take the first steps. People from both sides of the testability debate must be involved.

In the meantime, journal editors and publishers could assign speculative work to other research categories - such as mathematical rather than physical cosmology - according to its potential testability. And the domination of some physics departments and institutes by such activities could be rethought ${ }^{1,2}$.

The imprimatur of science should be awarded only to a theory that is testable. Only then can we defend science from attack.

George Ellis is professor emeritus of applied mathematics at the University of Cape Town, South Africa. Joe Silk is professor of physics at the Paris Institute of Astrophysics, France, and at Johns Hopkins University in Baltimore, Maryland, USA.

e-mails: george.ellis@uct.ac.za; silk@iap.fr

1. Woit, P. Not Even Wrong (Cape, 2006).

2. Smolin, L. The Trouble with Physics (Penguin, 2006).

3. Baggott, J. Farewell to Reality (Constable, 2013).

4. Ellis, G. F. R. Sci. Am. 305, 38-43 (2011); available at http://go.nature.com/27p6oe.

5. Steinhardt, P. Nature 510, 9 (2014)

6. Dawid, R. Phil. Sci. 73, 298-332 (2007).

7. Ellis, G. F. R. Gen. Rel. Grav. 46, 1619 (2014).

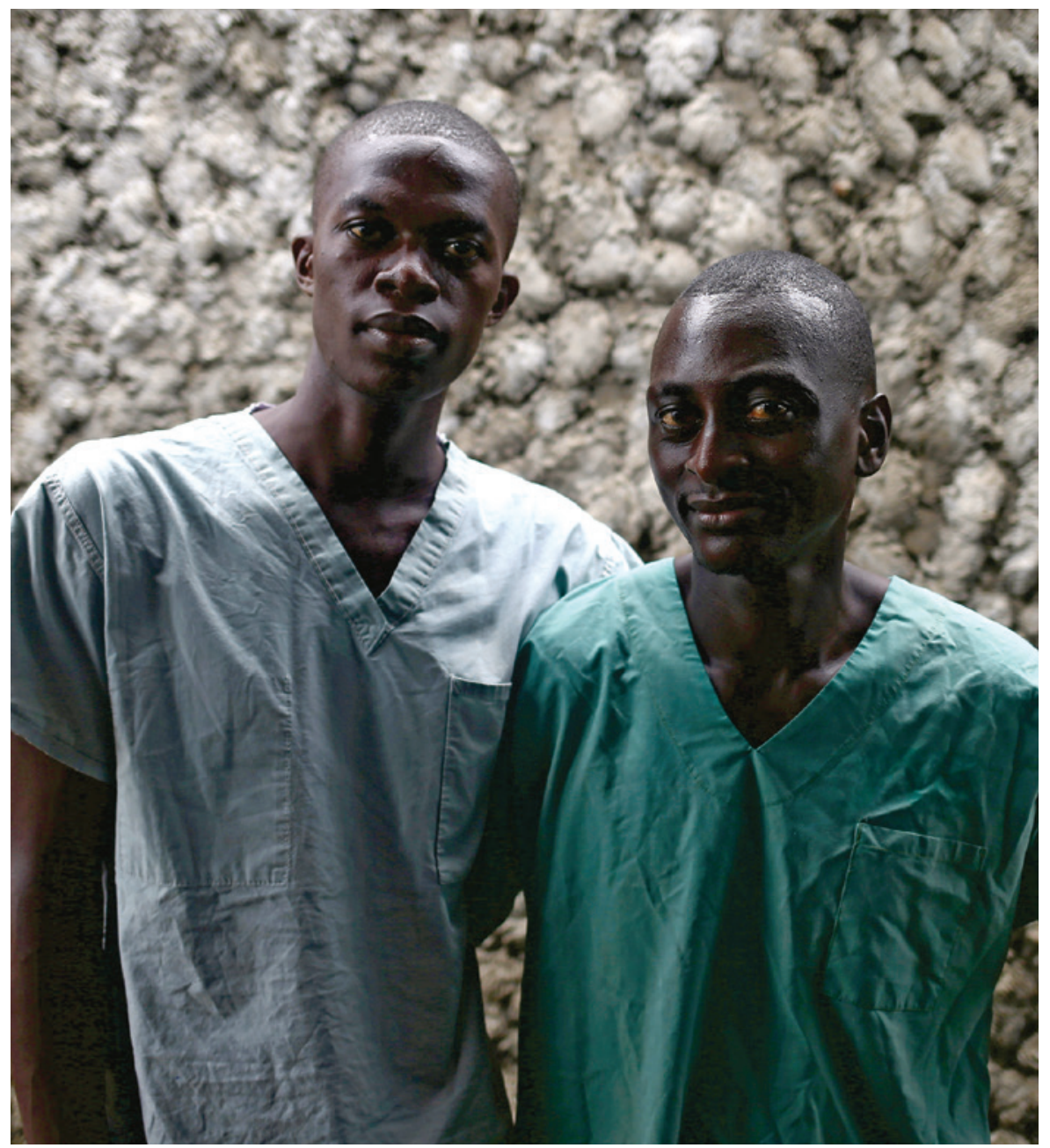

Ebola survivors Zaizay Mulbah (left), a former money changer, and Mark Jerry, previously a delivery driver, are working as nurses' assistants at a Liberian Ebola centre.

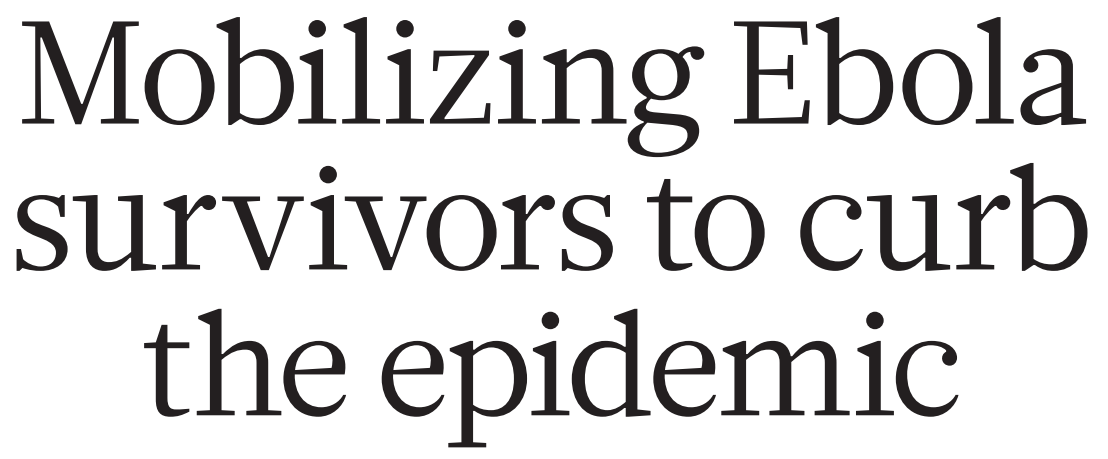

Scaling up the recruitment of individuals who have recovered from infection deserves urgent consideration, argue Joshua M. Epstein, Lauren M. Sauer and colleagues.

$\mathrm{M}$

ultiple governments and nongovernmental organizations have called on health-care personnel the world over to help control West Africa's Ebola outbreak; these include Médecins Sans Frontières (MSF), the World Health Organization (WHO) and United Nations children's charity UNICEF. But the demand for labour far exceeds the supply ${ }^{1}$ UN estimates, which may be low, suggest that approximately 5,000 international medical, training and support personnel are needed in the coming months.

While foreign assistance must continue, a nascent local strategy is a candidate for broad adoption. We call it MORE, for 


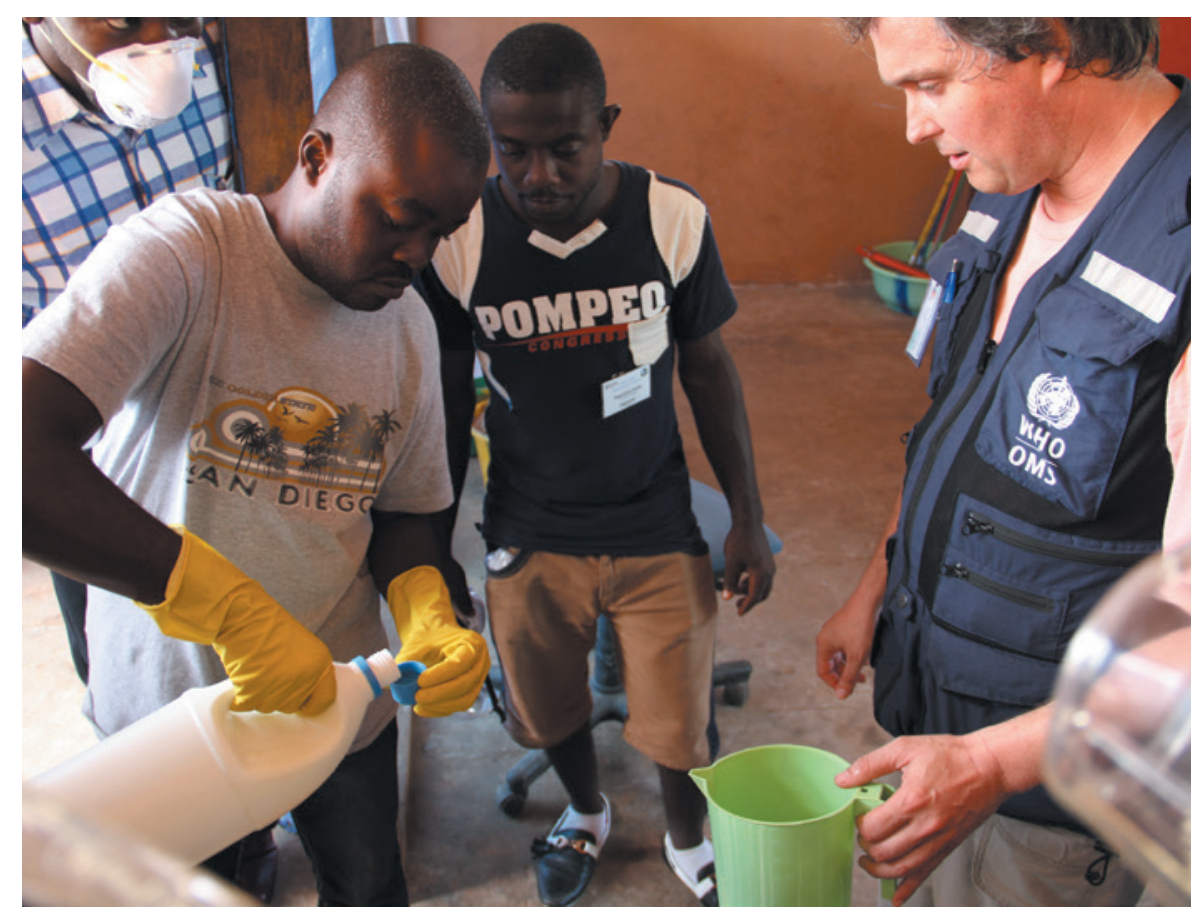

Ebola survivors are assisting in World Health Organization response efforts, which could be expanded.

> MObilization of REcovered individuals. The idea is simple: those who have recovered from Ebola could be engaged to reduce transmission, helping to bring the epidemic under control.

Examples of the approach can be seen in Sierra Leone, Guinea and Liberia. For instance, the UN is training survivors to support children who have had contact with infected individuals and are within Ebola's 21-day incubation window (the time it takes to develop symptoms after being infected with the virus). MSF is similarly employing survivors to work in their Ebola treatment units in Guinea and Liberia.

There are uncertainties about the ultimate size of this cadre and, crucially, about the immunity of recovered responders to reinfection, both immediately and in the longer term (because immunity may wane). Nonetheless, the potential of MORE to shift the epidemic's dynamics makes its consideration imperative.

\section{RECOVERED RESPONDERS}

So far, Ebola has infected an estimated 16,000 individuals in Liberia, Sierra Leone and Guinea. Current estimates suggest that in West Africa, roughly 50\% of people who contract Ebola will die ${ }^{2}$. This would leave a substantial pool of survivors, totalling perhaps 8,000 people by the end of the year. In the longer term, this could prove to be a much larger number. Indeed, the larger the epidemic, the bigger this pool becomes.

The worst-case projections of the US Centers for Disease Control and Prevention, for Sierra Leone and Liberia only, range from 500,000 to 1.4 million cases of Ebola by January 2015 (ref.3). Owing to various methodological limitations (set forth earlier by one of us, J.M.E., see go.nature.com/86kpyw), these projections are proving to be much too high. But even if the lower of these estimates turns out to be an order of magnitude too high, there could ultimately be 50,000 cases. If $50 \%$ survive, this is a pool of 25,000 . If we assume that $75 \%$ of survivors would be too young, too old, too ill or too traumatized to be recruited, the available cadre could still number in the thousands (see go.nature. com/kbx4el).
In classical epidemiology, susceptible people ( $S$ ) bump into infected ones $(I)$ as in a perfectly mixed bowl. That is, if $\beta$ is the transmission probability per contact between these pools, the epidemic grows at rate $\beta S I$.

To model the impact of the MObilization of REcovered individuals (MORE) strategy, we let $Z_{t}$ denote the recovered proportion of the population $t$ days into the epidemic, and $k$ denote the fraction of recovered people who are deployed to reduce transmission. This multiplies the classical growth rate above by $\left(1-k Z_{t}\right)$, which one might interpret as reducing $\beta$.

The reproductive number, $R_{t}$, is the average number of primary infections produced by a single infected individual
There are limited confirmatory data on protective immunity to Ebola in humans. But researchers generally agree that the evidence is pointing towards survivors being immune to reinfection. Thus far, there has not been a single reported case of a person who recovered from Zaire ebolavirus (the lineage of the current outbreak) becoming reinfected. This, and evidence from animal studies, suggests that people may have protective immunity following recovery.

\section{RISK LEVEL}

At worst, recovered responders would have the same level of risk as the general population, in which case they would need to use the same personal protective equipment (PPE) as other responders. At best, they would have high protection through conferred immunity.

In the latter event, recovered responders could operate with much less onerous PPE than current health-care workers. They would require only the training and protective equipment (medical gloves, face shield and goggles) used to minimize the transmission of more familiar blood-borne pathogens such as HIV. This would allow them to have much more extensive contact with patients than Ebola PPE normally affords. Generally, providers in full Ebola PPE work only two-hour shifts to avoid overheating (see go.nature.com/hsk4v5).

Recovered individuals can be trained to perform many important response functions (see 'Responder roles'). Some of these are beyond palliative, and may have a direct impact on disease transmission, changing the course of the epidemic itself. Such activities include isolating suspected patients from uninfected community members

\section{EBOLA CONTROL \\ Reversing the epidemic}

dropped into the population on day $t$. If $R_{t}>1$ the epidemic is growing, whereas if $R_{t}<1$, it is declining. So, $R_{t}=1$ is the epidemic threshold. Letting $R_{t}^{M}$ and $R_{t}^{C}$ denote, respectively, the reproductive numbers in the MORE and classical models (including deaths), it follows that:

$$
R_{t}^{M}=R_{t}^{C}\left(1-k Z_{t}\right)
$$

So, if $k$ (the mobilized fraction of the recovered) exceeds zero, MORE reduces the reproductive number. And crucially, if $R_{t}=1$ or is hovering above it, mobilized survivors could tip the epidemic into fading out. More-realistic models, with social networks rather than perfect mixing, could reveal stronger effects. 
